\title{
Genotipos de aislados de campo de Brucella abortus de distintas regiones geográficas de Chile ${ }^{\#}$
}

\author{
Genotypes of Brucella abortus field isolates from different \\ geographical regions of Chile \\ M Mancilla ${ }^{\mathrm{a}}$, M Villarroel ${ }^{\mathrm{b}}$, ME Saldías ${ }^{\mathrm{b}}, \mathrm{J} \mathrm{Soto}^{\mathrm{b}}$, AM Zárraga $^{\mathrm{a}^{*}}$ \\ anstituto de Bioquímica, Facultad de Ciencias, Universidad Austral de Chile,Valdivia, Chile.

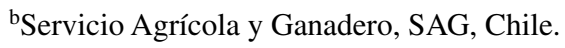

\begin{abstract}
SUMMARY
Bovine brucellosis is an endemic, zoonotic disease of high economic impact. The genetic identification of the prevalent Brucella abortus strains, the pathogen, is key to pursue further epidemiological strategies for disease control. The insertion sequence IS711 has been used as genetic marker to differentiate among Brucella species, members of the same specie and within the same biovar. We have analyzed the IS711- RFLP pattern for 46 B. abortus isolates, collected during the period of 1997-2005 from 16 different geographical areas of Chile. All isolates were previously identified by conventional techniques as B. abortus biovar 1. Of these, $87 \%$ shared the same IS711 DNA profile, while an $8.7 \%$ corresponded to the pattern of RB51 vaccine strain. We report the finding of two new strains, not differentiated by AMOS PCR, which showed unreported patterns of IS711-RFLP.
\end{abstract}

Palabras clave: Brucella abortus, genotipificación, PCR AMOS, IS711.

Key words: Brucella abortus, genotyping, AMOS PCR, IS711.

\section{INTRODUCCION}

Las bacterias pertenecientes al género Brucella son responsables de una de las más importantes infecciones zoonóticas a nivel mundial, causante de aborto infeccioso en animales y de la enfermedad febril en humanos, también conocida como fiebre ondulante. Este patógeno se caracteriza por ser un bacilo gram negativo, intracelular facultativo, capaz de infectar al macrófago del hospedero en los cuales se multiplica y disemina en forma sistémica (Gorvel y Moreno 2002). La brucelosis animal es un problema sanitario que genera graves pérdidas económicas y limita el comercio internacional pecuario. En países desarrollados como Australia y Reino Unido se ha logrado erradicar la enfermedad aplicando estrictas medidas de control e higiene (Boschiroli y col 2001). Sin embargo, aún se presenta en forma endémica y con importante impacto económico en Africa, Medio Oriente, Asia, algunos países del Mediterráneo y Sudamérica ${ }^{1}$. En Chile, la enfermedad está diseminada en todas las regiones productoras de ganado bovino, ubicándose preferentemente entre las VII y X Regiones, siendo esta última donde se concentra la mayor masa ganadera (Lopetegui 2005, Rivera y col 2002).

\footnotetext{
Aceptado: 26.09.2007.

\# Financiamiento DID-UACh D-2005-17 y FONDEF D02I1111.

* Casilla 567, Valdivia, Chile; anamariazarraga@uach.cl

1 http://www.unav.es/microbiol/pagina_8.html
}

A la fecha, se conocen seis especies de Brucella, de las cuales sólo B. abortus, B. melitensis, B. suis y B. canis son zoonóticas y se transmiten directa o indirectamente a los humanos por el bovino, cabra, cerdo y perro, respectivamente. Para la situación sanitaria bovina, la brucelosis más relevante es la producida por Brucella abortus. Las estrategias de control contemplan la inmunización con la cepa vacuna RB51. Esta cepa presenta la ventaja de no interferir con el diagnóstico serológico, a diferencia de la cepa S19, utilizada con anterioridad, la cual induce la aparición de anticuerpos en suero y en leche (Ramírez y col 2002, Rivera y col 2002). Se han reportado casos esporádicos de brucelosis, incluyendo zoonosis (Villarroel y col 2000, Ashford y col 2004, Moriyon y col 2004), los cuales se han asociado al uso de la cepa vacuna RB51 (Ewalt y Bricker 2000). Ambas vacunas se distinguen mediante ensayos de susceptibilidad de los cutivos utilizando eritritol para la cepa $\mathrm{S} 19$ y rifampicina para la cepa RB51 (Bricker y Halling 1995).

En los últimos años se han descrito varias estrategias moleculares para la identificación y diferenciación entre biovares y especies del género Brucella (Bricker 2002). Entre estas, la técnica de la reacción de la polimerasa en cadena o PCR denominado AMOS (Bricker y Halling 1994) permite diferenciar entre B. abortus, B. melitensis, B. ovis y B. suis. Esta metodología ha sido modificada para distinguir además entre las cepas de B. abortus 2308 y la cepa vacuna RB51 (Bricker y Halling 1995, Ewalt y Bricker 2000). En este trabajo se utilizó el análisis de 
fingerprint IS711 o IS711-RFLP para discriminar entre cepas chilenas del género Brucella agrupadas en un mismo biovar (Marianelli y col 2003, Ocampo-Sosa y col 2005). El polimorfismo reportado a la fecha respecto al número de copias de esta secuencia y su distribución en el genoma de Brucella le otorga mayor capacidad discriminatoria a este marcador genético frente al PCR dirigido a loci conservados para cada especie. Los resultados indican una baja variabilidad genética en la población chilena de B. abortus, identificándose dos perfiles nuevos de IS711RFLP para esta especie, no descritos previamente.

\section{MATERIAL Y METODOS}

\section{CEPAS BACTERIANAS Y EXTRACCION DE ADN GENOMICO}

Se estudiaron 46 aislados de campo, 21 recuperados de muestras de leche y 25 de restos fetales, provenientes de cinco regiones y 16 localidades de Chile, recolectados entre 1997-2005 (cuadro 1). Estos aislados, junto con las cepas de referencia 544 y vacunas S19 y RB51, se obtuvieron del Laboratorio Central del Servicio Agrícola y Ganadero (SAG) Lo Aguirre, Santiago, y del Laboratorio Regional de Osorno. La cepa 2308 se obtuvo de la Universidad de Concepción. Las bacterias fueron cultivadas a $37{ }^{\circ} \mathrm{C}$ durante 48-72 h en agar Brucella (BBL, Beckton-Dickinson), en condiciones de microaerofilia generadas con el kit Genbag (Biomerieaux) y mantenidas a $-70{ }^{\circ} \mathrm{C}$, en glicerol $20 \%$.

Todos los aislados fueron previamente tipificados como B. abortus biovar 1 utilizando criterios bioquímicos, serológicos, susceptibilidad a antibióticos y fagos. Para la extracción de ADN genómico, las bacterias fueron recolectadas desde placas de agar Brucella, se lisaron por tratamiento con lisozima-SDS-proteinasa K (Sambrook y col 1989) seguido de extracción con solventes orgánicos para remover posibles interferentes. Finalmente, el ADN fue precipitado con isopropanol, lavado con etanol, resuspendido en agua y mantenido a $-20^{\circ} \mathrm{C}$. Previo a la cuantificación espectrofotométrica, las muestras se trataron por $1 \mathrm{~h}$ a $37^{\circ} \mathrm{C}$ con RNAsa libre de DNAsa (10 $\mu \mathrm{g} / \mathrm{ml})$. Una estrategia alternativa de extracción consistió en la inactivación por calor $\left(80^{\circ} \mathrm{C}\right.$ por $\left.30 \mathrm{~min}\right)$ de una suspensión de bacterias en suero fisiológico, seguida de centrifugación y recuperación del sobrenadante, la cual se utilizó como templado de la reacción de PCR.

\section{PCR AMOS}

El test de PCR AMOS se utilizó para la identificación de las cepas (Bricker y Halling 1994, Bricker y Halling 1995). La reacción se llevó a cabo en un volumen final de $25 \mu \mathrm{l}$ conteniendo $0,2 \mu \mathrm{M}$ de cada partidor (de 5, a 3': BA, GACGAACGGAATTTTTCCAATCCC; IS 711, TGCCGATCACTTAAGGGCCTTCAT; RB 51, CCCCGGAAGATATGCTTCGATCC; Eri-1, GCGCCGCGAAGAACTTATCAA; Eri-2,
CGCCATGTTAGCGGCGGTGA) 2,5 $\mu$ de buffer 10x de enzima, $2 \mathrm{mM}$ de $\mathrm{MgCl}_{2}, 0,4 \mathrm{mM}$ de cada dNTP, 1 $\mathrm{U}$ de Taq polimerasa (Invitrogen), y $10 \mathrm{ng}$ de ADN. El programa de amplificación comenzó con una etapa de desnaturación a $95^{\circ} \mathrm{C}$ por 5 min, seguido de 30 ciclos de desnaturación a $95^{\circ} \mathrm{C}$ por $1 \mathrm{~min}$, apareamiento a $60^{\circ} \mathrm{C}$ 1 min y extensión a $72{ }^{\circ} \mathrm{C}$ por 2 min, para finalizar con un ciclo de extensión a $72{ }^{\circ} \mathrm{C}$ por 7 min (termociclador, GeneAmp 9700, Applied Biosystem). Los productos de amplificación se visualizaron en geles de agarosa al 1,5\% teñido con bromuro de etidio, $0,5 \mu \mathrm{g} / \mathrm{ml}$ sobre pantalla transiluminadora UV. Las imágenes se capturaron con sistema de fotodocumentación (Kodak 1D, versión 1.0, Kodak).

\section{IS711-RFLP}

Este procedimiento se realizó de acuerdo al protocolo descrito previamente (Ocampo-Sosa y col 2005), con algunas modificaciones. El ADN genómico de cada aislado $(1-2 \mu \mathrm{g})$ fue digerido toda la noche a $37{ }^{\circ} \mathrm{C}$ con las enzimas de restricción AvaI y ClaI (Fermentas). Los fragmentos de restricción se resolvieron en geles de agarosa al $1,0 \%$ y se transfirieron por capilaridad, en condiciones alcalinas por $16 \mathrm{hrs}$ a temperatura ambiente a membranas de nylon (GeneScreen, GeneLab). Posteriormente, el ADN se fijó a la membrana por calor a $80{ }^{\circ} \mathrm{C}$ por $30 \mathrm{~min}$. La sonda de $967 \mathrm{bp}$, conteniendo el elemento IS711, fue generada por PCR usando como templado el ADN genómico de la cepa vacuna $B$. abortus RB51. La mezcla de reacción $(50 \mu \mathrm{l})$ consistió de $0,2 \mu \mathrm{M}$ de cada partidor (711u, CACAAGACTGCGTTGCCGACAGA; 711d, CATATGATGGGACCAAACACCTAGGG), 5,0 $\mu l$ de buffer 10x de enzima, $2 \mathrm{mM}$ de $\mathrm{MgCl}_{2}, 0,4 \mathrm{mM}$ de cada dNTP, $1 \mathrm{U}$ de Taq polimerasa (Invitrogen) y $10 \mathrm{ng}$ de ADN genómico bacteriano. El programa de amplificación se inició denaturando el ADN a $95{ }^{\circ} \mathrm{C}$ por $5 \mathrm{~min}$, seguido de 30 ciclos de desnaturación a $95^{\circ} \mathrm{C}$ por $1 \mathrm{~min}$, apareamiento a $60^{\circ} \mathrm{C}$, extensión a $72{ }^{\circ} \mathrm{C}$ por 1 min para finalizar con un ciclo de extensión a $72^{\circ}$ por $5 \mathrm{~min}$. La sonda se purificó a partir de geles de agarosa, utilizando el kit QIAEXII, siguiendo las instrucciones del fabricante (Qiagen). Como marcador de masa molecular, se utilizó el estándar de ADN fago $\lambda$ /HindIII (Fermentas) marcado con biotina. El ADN transferido fue hibridado con un $\mu \mathrm{g}$ de sonda biotinilada (kit Bio-Nick, Invitrogen) e incubado a $42{ }^{\circ} \mathrm{C}$ por $2 \mathrm{~h}$ en equipo para hibridación (Hybridiser, HB-1D, Techne), previo bloqueo por $3 \mathrm{~h}$ con solución de prehibridación conteniendo ADN de espermio de salmón a $100 \mu \mathrm{g} / \mathrm{ml}$ (Invitrogen). Posteriormente, la señal de hibridación fue revelada por quimioluminiscencia utilizando el kit DNA Detector, siguiendo las instrucciones del fabricante (KPL). La señal de hibridación se registró en film de autorradiografía por exposición durante 30 min a temperatura ambiente. Para la determinación de los distintos tipos de perfiles o clusters de IS711-RFLP, las 
Cuadro 1. Antecedentes de los aislados de Brucella abortus utilizados en este estudio. Código: numeración arbitraria interna. Año: año de aislamiento. Muestra: origen biológico del aislado. Localidad y Región: zonas geográficas de origen de los aislados.

Information on Brucella abortus isolates used in this study. Código: arbitrary identification number. Año: year of isolation. Muestra: isolate biological source. Localidad and Región: geographical origin of the isolates

\begin{tabular}{|c|c|c|c|c|}
\hline Código & Año & Muestra & Localidad & Región \\
\hline B01 & 2004 & Leche & Talagante & $\mathrm{RM}$ \\
\hline В02 & 2004 & Leche & Talagante & $\mathrm{RM}$ \\
\hline B03 & 2004 & Leche & Talagante & $\mathrm{RM}$ \\
\hline B04 & 2004 & Leche & Talagante & $\mathrm{RM}$ \\
\hline B05 & 2004 & Leche & Talagante & $\mathrm{RM}$ \\
\hline B06 & 2004 & Leche & Talagante & $\mathrm{RM}$ \\
\hline В07 & 2005 & Feto & Osorno & $\mathrm{X}$ \\
\hline B08 & 2005 & Feto & Puyehue & $X$ \\
\hline B10 & 2005 & Feto & Puerto Montt & $X$ \\
\hline B11 & 2004 & Feto & Río Negro & $\mathrm{X}$ \\
\hline B12 & 2004 & Leche & Río Bueno & $X$ \\
\hline B13 & 1999 & Feto & Río Bueno & $\mathrm{X}$ \\
\hline B14 & 2000 & Feto & Ultima Esperanza & XII \\
\hline B15 & 2000 & Feto & Puerto Octay & $\mathrm{X}$ \\
\hline B16 & 2002 & Feto & Osorno & $X$ \\
\hline B17 & 2003 & Leche & San Carlos & VIII \\
\hline B18 & 2004 & Leche & Puerto Montt & $X$ \\
\hline B19 & 2004 & Leche & Puerto Octay & $X$ \\
\hline B20 & 2003 & Leche & San Carlos & VIII \\
\hline B21 & 2004 & Leche & Freire & IX \\
\hline B22 & 2004 & Leche & La Unión & $\mathrm{X}$ \\
\hline B23 & 2003 & Leche & San Carlos & VIII \\
\hline B24 & 2004 & Leche & Puerto Octay & $X$ \\
\hline B25 & 2003 & Leche & Río Negro & $X$ \\
\hline B26 & 2003 & Leche & Bulnes & VIII \\
\hline B27 & 2003 & Leche & Bulnes & VIII \\
\hline B28 & 2003 & Leche & Río Negro & $\mathrm{X}$ \\
\hline B29 & 2003 & Leche & San Carlos & VIII \\
\hline B30 & 2004 & Leche & Puerto Octay & $X$ \\
\hline B31 & 1997 & Feto & Paillaco & $X$ \\
\hline B32 & 1997 & Feto & Osorno & $X$ \\
\hline B33 & 1997 & Feto & Tacamo & $X$ \\
\hline B34 & 1997 & Feto & Río Bueno & $X$ \\
\hline B35 & 1997 & Feto & Purranque & $X$ \\
\hline B36 & 1997 & Feto & Valdivia & $X$ \\
\hline B37 & 1997 & Feto & Puerto Montt & $\mathrm{X}$ \\
\hline B38 & 1997 & Feto & Purranque & $X$ \\
\hline B39 & 1997 & Feto & Purranque & $X$ \\
\hline B40 & 1997 & Feto & Puerto Montt & $X$ \\
\hline B41 & 1997 & Feto & Río Negro & $X$ \\
\hline B42 & 1997 & Feto & Puyehue & $X$ \\
\hline B43 & 1998 & Feto & Río Bueno & $X$ \\
\hline B45 & 1998 & Feto & Osorno & $X$ \\
\hline B46 & 1998 & Feto & Purranque & $X$ \\
\hline B47 & 1998 & Feto & Río Negro & $X$ \\
\hline B48 & 1998 & Feto & Río Negro & $X$ \\
\hline
\end{tabular}


imágenes de los films fueron capturadas por sistema de fotodocumentación y analizadas con el programa Gelcompar II (versión 3.5, Applied Maths), utilizando el algoritmo de comparación UPGMA, modo bandas diferentes con una tolerancia ajustada al $1,5 \%$.

\section{RESULTADOS Y DISCUSION}

Existe una amplia gama de métodos moleculares descritos para la tipificación de especies del género Brucella. Algunos se aplican para la identificación de género, los cuales son elegibles preferentemente para el diagnóstico de brucelosis humana y en microbiología de alimentos (Bricker 2002). Sin embargo, para establecer las causas de brotes y diseminación de la enfermedad es importante establecer la identidad de las variantes genéticas de la especie Brucella, ya que todas las cepas patógenas de este género son potencialmente diseminadoras (Bricker 2004). En Chile, los programas de vigilancia y control están dirigidos a erradicar las infecciones causadas por B. abortus, única especie identificada a la fecha como agente etiológico de los casos de brucelosis bovina. El programa nacional utiliza la cepa RB51 como vacuna, acompañada de la aplicación de adecuadas prácticas de manejo del rebaño, observándose una prevalencia informada de $B$. abortus en los rebaños del país de un 1\% (Lopetegui 2005).

En este estudio, la identidad del ADN genómico de todos los aislados de campo fue confirmada por PCR AMOS. Los aislados de B. abortus, independiente del tipo de muestra, origen y localización geográfica (cuadro 1), generaron una banda de 498 bp, característica de esta especie (figura 1, sección c). Todos los aislados presentaron además un producto de amplificación de $178 \mathrm{bp}$, correspondiente a la amplificación del locus ery. En el grupo en estudio no se encontraron perfiles coincidentes con el de la cepa S19, cuyo genoma presenta una deleción de 702 bp que comprende este locus. Los aislados de B. abortus B07, B08, B42 y B43, resistentes a rifampicina, mostraron una banda adicional de $364 \mathrm{bp}$, perfil concordante con el de las cepas de referencia 2308 y RB51. Para discriminar entre las cepas de $B$. abortus, se utilizó la técnica IS711-RFLP (figura 1, sección b). El tamaño de los fragmentos conteniendo la secuencia IS711 osciló entre 1,1-7,2 kb. (figura $1 *$ ). Cada fragmento contiene una copia del IS711, dado que esta secuencia carece de sitios de corte para las enzimas de restricción AvaI y ClaI. El análisis con el programa Gelcompar II agrupó los perfiles en cuatro grupos sin incluir la cepa 2308 (figura 1, sección a). El perfil encontrado en la mayoría de los aislados de B. abortus coincide con el de la cepa de referencia 544 biovar 1 (arbitrariamente designado perfil C). Este perfil se caracteriza por la presencia de siete copias de IS711, concordando con el número de copias encontrado en el genoma anotado de B. abortus. Las cepas de origen de estos perfiles se aislaron de muestras de leche y restos fetales de las Regiones Metropolitana y XII $(n=40)$. Cabe destacar que la cepa de B. abortus biovar
1 fue responsable del $85 \%$ de los casos de brucelosis en EE.UU el año 2003 (Bricker 2004). Los aislados B07, B08, B42 y B43 presentaron el segundo genotipo más frecuente caracterizado por la presencia de 8 copias de IS711 (genotipo D), coincidiendo con el de la cepa vacuna RB51. Estos aislados se obtuvieron a partir de muestras de restos fetales de bovinos provenientes de la X Región. Este perfil se diferencia de la cepa 2308 por la presencia de una copia adicional de IS711, localizada en un fragmento de restricción de 1,5 kb. Destacan de este estudio los aislados B12 y B16, obtenidos a partir de leche y restos fetales de muestras de la X Región. La figura 2 muestra los distintos perfiles IS711 identificados, representativos de las cepas chilenas. Las cepas cuyos perfiles se indican en los carriles 1 y 2 corresponden a las cepas B16 (perfil A) y B12 (perfil B). Estas cepas presentaron repetidamente perfiles únicos no descritos previamente. El genoma de estas cepas es concordante con el genotipo $\mathrm{C}$ mayoritario, excepto por la presencia de una copia de IS711 contenida en un fragmento de restricción de tamaño $6,6 \mathrm{~kb}$ para la cepa B12 y de 5,5 kb en la cepa B16 (figura 2, carriles B y A, respectivamente). Se puede especular que estas nuevas variantes genéticas serían el resultado de eventos de transposición replicativa recientes, ya que no tenemos conocimiento que se hayan reportado con anterioridad. Cabe destacar que la cepa B16 es fenotípicamente distinta al resto ya que carece de la actividad ureasa. La posibilidad de que la secuencia IS711 presente en la banda adicional esté interfiriendo directa o indirectamente en la expresión de esta enzima multimérica, ureasa, (Sangari y col 2007) está siendo investigada. De acuerdo a los antecedentes de origen de las muestras, las cepas B12 y B16 parecieran no estar asociadas a diferencias en la capacidad patogénica respecto a la cepa silvestre.

En conclusión, los 46 aislados de B. abortus biovar 1 incluidas en este estudio se agruparon en cuatro perfiles IS711 distintos. Grupo 1: genotipo C, de mayor abundancia (87\%), caracterizado por siete copias de IS711, idéntico al perfil de la cepa 544. Grupo 2: perfil caracterizado por presentar ocho copias de IS711 (genotipo D). Este es el segundo genotipo en abundancia $(8,7 \%)$ e incluye a la cepa RB51, la cual deriva de la cepa 2308 (Vemulapalli y col 1999). Grupo 3: genotipo A correspondiente a la cepa B16 ( $n=1)$ con ocho copias de IS711. Grupo 4: representado por el genotipo B que identifica a la cepa B12 $(n=1)$ con ocho copias de IS711. La técnica de RFLP-IS711, a diferencias de la técnica de PCR AMOS, discriminó entre cepas de B. abortus, a pesar de la limitada diversidad genética reportada para estas bacterias (Vizcaino y col 2000, Gandara y col 2001). La homogeneidad genética observada en la mayoría de los aislados (perfil C) indica la necesidad de complementar estos estudios utilizando las secuencias VNTR (Número Variable de Repeticiones en Tandem) para el género Brucella (Bricker y col 2003, Le Fleche y col 2006) como blanco génico. La naturaleza multilocus de estas secuencias entrega una visión más 


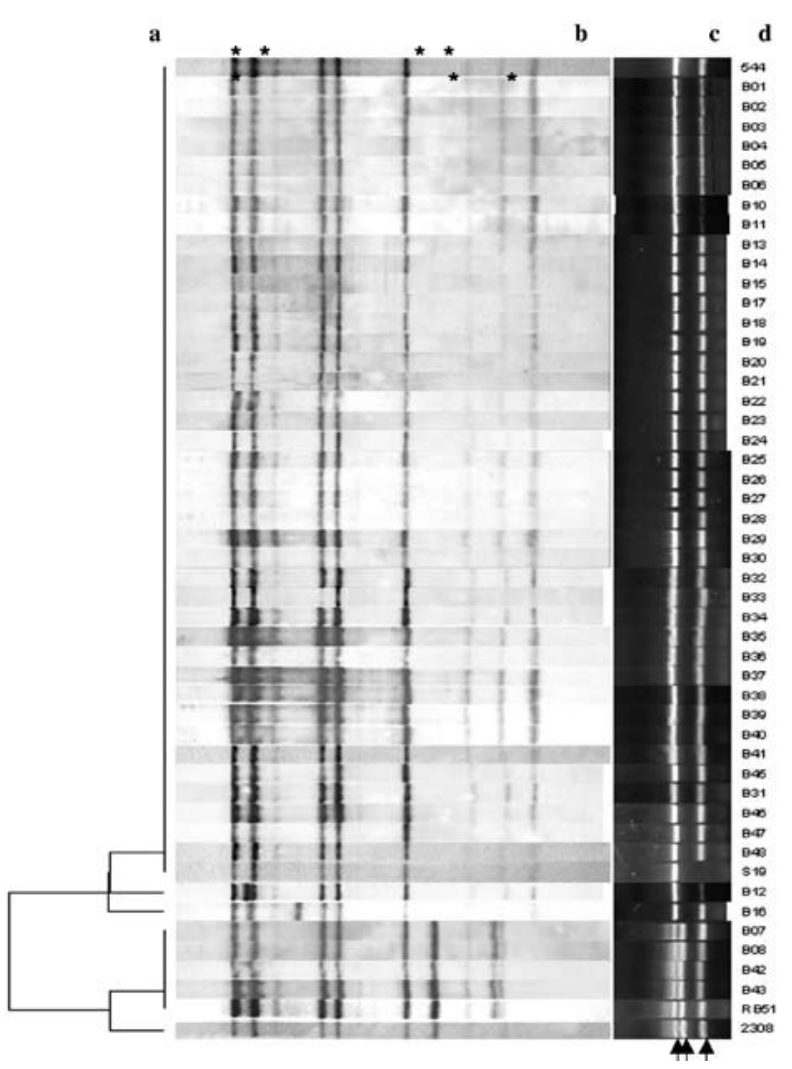

Figura 1. Caracterización genética de aislados chilenos de B. abortus.

a, Dendrograma; b, patrones de IS711-RFLP, (*): fragmentos IS711; c, PCR AMOS abreviado; Flechas, productos de PCR 498, 364 y 178 pares de bases, de izquierda a derecha; $d$, código de cada aislado. Cepas de referencia de B. abortus biovar 1: 544, 2308, S19 y RB51.

Genetic characterization of Chilean Brucella abortus isolates. a, Dendrogram; b, IS711-RFLP patterns, (*): IS711 fragments; c, Abbreviated AMOS PCR; Arrows (left to right): PCR products 498, 364 and 178 base pairs; d, Isolate identification number; B. abortus biovar 1 reference strains: 544, 2308, S19 and RB51.

precisa sobre la frecuencia y distribución geográfica de las variantes genéticas de $B$. abortus, requisito indispensable para trazar las rutas de diseminación del patógeno y diferenciar episodios de infección exógena de brotes de la enfermedad.

\section{RESUMEN}

La brucelosis bovina es una enfermedad zoonótica, endémica de alto impacto económico. La identificación genética de las cepas prevalentes de Brucella abortus, el patógeno, es clave para establecer estrategias epidemiológicas de control de la enfermedad. La secuencia de inserción IS711 ha sido utilizada como un marcador genético para diferenciar entre especies de Brucella, miembros de una misma especie y dentro de un mismo biovar. Hemos analizado los perfiles de IS711-RFLP de 46 aislados de B. abortus, recolectados durante el periodo 1997-2005, provenientes de 16 áreas geográficas diferentes de Chile. Todos los aislados fueron previamente identificados como B. abortus biovar 1, utilizando las técnicas convencionales. De estos, el $87 \%$ compartieron el mismo perfil de IS711-RFLP, mientras que el 8,7\% correspondió al patrón de la cepa vacuna RB51. En este trabajo se informa el hallazgo

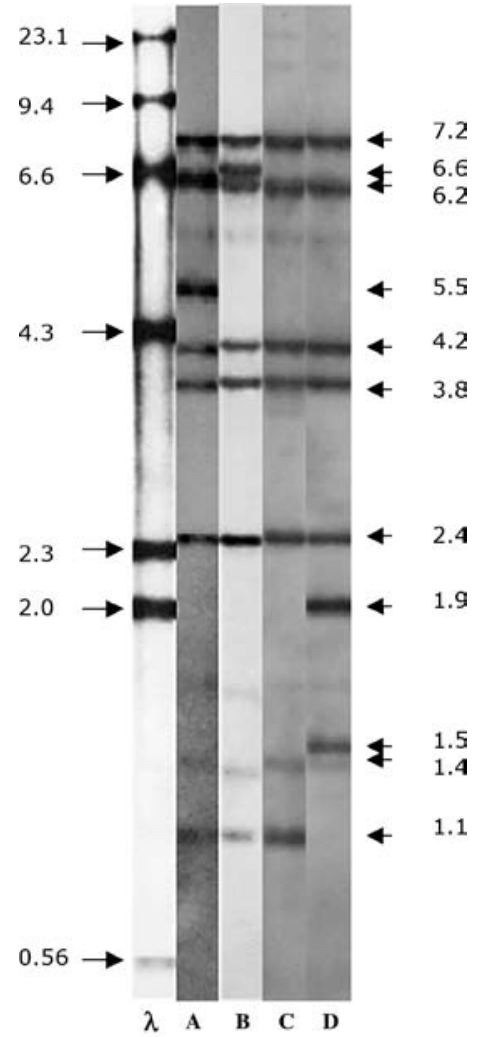

Figura 2. Perfiles IS711-RFLP representativos de cepas chilenas de $B$. abortus. $\lambda$ : marcador ADN fago.

Representative IS711-RFLP patterns of Chilean B. abortus strains. $\lambda$ : biotinylated Lambda phage/HindIII DNA as marker. Scale in kilobases. Lane A: B16 strain. Lane B: B12 strain. Lane C: 544 strain. Lane D: RB51 strain.

de dos cepas indistinguibles por PCR AMOS con perfiles nuevos de IS711-RFLP, no reportados previamente.

\section{AGRADECIMIENTOS}

Agradecemos al Dr. Angel Oñate por facilitarnos la cepa 2308. A la Comisión Nacional de Ciencia y Tecnología, CONICYT, la cual otorgó beca de postgrado al Sr. Marcos Mancilla.

\section{REFERENCIAS}

Ashford DA, J di Pietra, J Lingappa, C Woods, H Noll, B Neville, R Weyant, SL Bragg, RA Spiegel, J Tappero, BA Perkins. 2004. Adverse events in humans associated with accidental exposure to the livestock brucellosis vaccine RB51. Vaccine 22, 3435-3439.

Boschiroli ML, V Foulongne, D O'Callaghan. 2001. Brucellosis: a worldwide zoonosis. Curr Opin Microbiol 4, 58-64.

Bricker BJ, SM Halling. 1994. Differentiation of Brucella abortus bv. 1,2, and 4, Brucella melitensis, Brucella ovis, and Brucella suis bv. 1 by PCR. J Clin Microbiol 32, 2660-2666.

Bricker BJ, SM Halling. 1995. Enhancement of the Brucella AMOS PCR assay for differentiation of Brucella abortus vaccine strains S19 and RB51. J Clin Microbiol 33, 1640-1642.

Bricker BJ. 2002. PCR as a diagnostic tool for brucellosis. Vet Microbiol 90, 435-446. 
Bricker BJ, DR Ewalt, SM Halling. 2003. Brucella 'HOOF-Prints': strain typing by multi-locus analysis of variable number tandem repeats (VNTRs). BMC Microbiol 3, 15.

Bricker BJ. 2004. Molecular diagnostics of animal brucellosis: A review of PCR-based assays and approaches. In: Lopez-Goni I, I Moriyon (eds). Brucella: Molecular and Cellular Biology. Horizon Bioscience, Pamplona, España, Pp 25-51.

Ewalt DR, BJ Bricker. 2000. Validation of the abbreviated Brucella AMOS PCR as a rapid screening method for differentiation of Brucella abortus field strain isolates and the vaccine strains, 19 and RB51. J Clin Microbiol 38, 3085-3086.

Gandara B, AL Merino, MA Rogel, E Martinez-Romero. 2001. Limited genetic diversity of Brucella spp. J Clin Microbiol 39, 235-240.

Gorvel JP, E Moreno. 2002. Brucella intracellular life: from invasion to intracellular replication. Vet Microbiol 90, 281-297.

Le Fleche P, I Jacques, M Grayon, S Al Dahouk, P Bouchon, F Denoeud, K Nockler, H Neubauer, LA Guilloteau, G Vergnaud. 2006. Evaluation and selection of tandem repeat loci for a Brucella MLVA typing assay. BMC Microbiol 6, 9.

Lopetegui P. 2005. Avances de la erradicación de la brucelosis bovina en Chile. Boletín Veterinario Oficial, BVO N³. Servicio Agrícola y Ganadero, Santiago, Chile, Pp 1-14.

Marianelli C, G La Rosa, F Ciuchini, M Muscillo, P Pasquali, R Adone. 2003. Genetic diversity at alkB locus in Brucella abortus. $J$ Vet Med B 50, 494-499.

Moriyon I, MJ Grillo, D Monreal, D Gonzalez, C Marin, I Lopez-Goni, RC Mainar-Jaime, E Moreno, JM Blasco. 2004. Rough vaccines in animal brucellosis: structural and genetic basis and present status. Vet Res 35, 1-38.
Ocampo-Sosa AA, J Aguero-Balbin, JM Garcia-Lobo. 2005. Development of a new PCR assay to identify Brucella abortus biovars 5, 6 and 9 and the new subgroup 3b of biovar 3. Vet Microbiol 110, 41-51.

Ramírez M, S Ernst, F Elvinger, A Rivera, C Rosenfeld. 2002. Respuesta serológica y tiempo de saneamiento en rebaños bovinos vacunados con Cepa 19 o Cepa RB-51; X Región, Chile. Arch Med Vet 34, 213-220.

Rivera SA, MC Ramirez, IP Lopetegui. 2002. Eradication of bovine brucellosis in the 10th Region de Los Lagos, Chile. Vet Microbiol 90, 45-53.

Sambrook J, E Fritsch, T Maniatis. 1989. Molecular cloning: a laboratory manual. Cold Springs Harbor Laboratory Press, New York, USA.

Sangari FJ, A Seoane, MC Rodriguez, J Aguero, JM Garcia Lobo. 2007. Characterization of the urease operon of Brucella abortus and assessment of its role in virulence of the bacterium. Infect Immun 75, 774-780.

Vemulapalli R, JR McQuiston, GG Schurig, N Sriranganathan, SM Halling, SM Boyle. 1999. Identification of an IS711 element interrupting the wboA gene of Brucella abortus vaccine strain RB51 and a PCR assay to distinguish strain RB51 from other Brucella species and strains. Clin Diagn Lab Immunol 6, 760-764.

Villarroel M, M Grell, R Sáenz. 2000. Reporte de primer caso humano de aislamiento y tipificación de Brucella abortus RB51. Arch Med Vet 32, 89-91

Vizcaino N, A Cloeckaert, J Verger, M Grayon, L Fernandez-Lago. 2000. DNA polymorphism in the genus Brucella. Microbes Infect 2, 1089-1100. 